\title{
Construct validity and age sensitivity of prospective memory
}

\author{
TIMOTHY A. SALTHOUSE, DIANE E. BERISH, and KAREN L. SIEDLECKI \\ University of Virginia, Charlottesville, Virginia
}

\begin{abstract}
We administered four prospective memory tasks to 330 adults between 18 and 89 years of age to investigate the relationship among the measures of performance in the four tasks, as well as the relationship of the prospective memory measures to age, other cognitive abilities, and noncognitive factors. The four prospective memory variables were found to exhibit both convergent and discriminant validity, indicating that prospective memory ability appears to represent a distinct dimension of individual differences. The prospective memory construct was significantly related to other cognitive abilities, such as executive functioning, fluid intelligence, episodic memory, and perceptual speed, but it was only weakly related to self-ratings of (primarily retrospective) memory and to personality traits. Although a substantial proportion of the age-related variance on the prospective memory construct was shared with other cognitive abilities, we also found some evidence of unique, statistically independent, age-related influences on prospective memory.
\end{abstract}

Prospective memory refers to remembering to do something in the future. Although studied only recently, it has become of particular interest to researchers on aging because it may be more important for many aspects of everyday functioning and the ability to live independently than retrospective memory, which concerns information or events from the past. A considerable literature on adult age differences in prospective memory has emerged in the past 10-15 years (e.g., see reviews in Cherry et al., 2001; Henry, MacLeod, Phillips, \& Crawford, 2004; Maylor, 1996b; Maylor, Darby, Logie, Della Sala, \& Smith, 2002). There seems to be a consensus that age differences favoring young adults are evident in time-based prospective memory tasks, in which the future action is dependent on an elapsed interval or a specific time, but inconsistent findings have been reported with respect to the existence and magnitude of age differences in measures of eventbased prospective memory, in which the future action is dependent on the occurrence of a particular event.

In addition to this uncertainty about age-related effects, it is also unclear whether variables from different tasks hypothesized to assess prospective memory represent a unitary construct, as implied by use of the same label for each. The following quotations illustrate the differences of opinion concerning the status of a prospective memory construct. On the one hand, Mäntylä (2003,

This research was supported by NIA Grant RO1 AG019627 to T.A.S. We thank Thomas Atkinson, Jessica Busch, Preeti Chauhan, Angelia Lincoln, LaMont Mitchell, Melissa Ozim, Cristina Rabaglia, and Sarah Shelley for their help in scheduling and testing participants, scoring, and data entry. Correspondence concerning this article should be addressed to T. A. Salthouse, Department of Psychology, University of Virginia, P.O. Box 400400, Charlottesville, VA 22904-4400 (e-mail: salthouse@virginia.edu). p. 23) characterized prospective memory as "a relatively well-defined domain of memory," and Cockburn and Smith (1991, p. P32) claimed that "prospective memory skills ... are qualitatively different from those used in retrospective memory." On the other hand, Craik and Kerr (1996, p. 227) raised the possibility that "prospective memory may be nothing more than a unifying label for a wide range of tasks involving the timely performance of planned actions," and Crowder (1996), Dobbs and Reeves (1996), Ellis (1996), and Roediger (1996) have all expressed similar reservations about whether measures from tasks postulated to assess prospective memory represent a single construct.

One way to resolve the issue of whether prospective memory is a coherent and distinct construct, at least with respect to patterns of individual differences, involves investigating construct validity with convergent and discriminant validity procedures. Convergent validity refers to whether different variables hypothesized to represent the same construct are related to one another, such that they covary across people as one would expect if they reflect the same dimension of individual differences. Discriminant validity refers to whether the variables represent something different from what is represented by other constructs, as manifested by relatively weak correlations with these other constructs.

Construct validity can therefore be investigated by administering two or more prospective memory tasks to the same individuals, in addition to tasks hypothesized to represent other theoretical constructs. Simultaneous consideration of several variables hypothesized to reflect prospective memory has the added advantage of improving assessment of the construct. That is, the most meaningful level of analysis is typically not a single variable from a particular task, regardless of the presumed purity 
or elegance of the task, because almost all variables incorporate some influences specific to the methods, materials, and measures in that task. One method of minimizing nonconstruct influences consists of simultaneously examining several variables, and then focusing on what those variables have in common. Not only does this analytical procedure serve to average out the specific influences on each variable, but the resulting assessment is more reliable because variance common to the different variables does not include measurement error (or else it could not be shared with other variables).

Only a few studies have been reported in which more than one event-based prospective memory task was administered to the same individuals. Furthermore, the results of those studies have been inconsistent, because moderate correlations were reported by Cockburn and Smith (1991) and Vogels, Dekker, Brouwer, and de Jong (2002), but low and generally nonsignificant correlations were reported by Chasteen, Park, and Schwarz (2001), Kidder, Park, Hertzog, and Morrell (1997), and Schmidt, Berg, and Deelman (2001). The lack of strong correlations among different measures of what is purportedly the same construct obviously raises questions about the convergent validity of a prospective memory construct.

Correlations of prospective memory measures with other cognitive variables have been reported in a number of studies. For example, significant relations with prospective memory variables have been reported with measures of verbal memory (e.g., Cherry et al., 2001; Graf, Uttl, \& Dixon, 2002; Groot, Wilson, Evans, \& Watson, 2002; Huppert, Johnson, \& Nickson, 2000; Reese \& Cherry, 2002; Uttl, Graf, Miller, \& Tuokko, 2001), working memory (e.g., Cherry \& LeCompte, 1999; Kidder et al., 1997; Park, Hertzog, Kidder, Morrell, \& Mayhorn, 1997; Reese \& Cherry, 2002; West \& Craik, 2001), processing speed (e.g., Graf et al., 2002; Groot et al., 2002; Martin \& Schumann-Hengsteler, 2001; Maylor 1993, 1996a; Uttl et al., 2001), nonverbal reasoning (Cockburn \& Smith, 1991; Groot et al., 2002; Maylor, 1996a), and vocabulary (e.g., Cherry \& LeCompte, 1999; Reese \& Cherry, 2002). Note that the existence of significant correlations with variables representing other constructs is potentially inconsistent with discriminant validity of the prospective memory construct.

Although the results from the studies cited thus far do not provide strong support for the construct validity of prospective memory, more favorable results were reported in two recent studies. Both studies conducted exploratory factor analyses and found that prospective memory variables loaded on different factors than variables from retrospective memory or other cognitive tasks (e.g., Maylor, Smith, Della Sala, \& Logie, 2002; Uttl et al. 2001).

Unfortunately, most of the studies mentioned in the preceding paragraphs share a number of limitations. For example, many had small samples (some with as few as 16 individuals in each age group) which leads to low power and the possible presence of a few extreme obser- vations that could greatly influence the magnitude of correlations with other variables, and sometimes prospective memory was assessed very coarsely with a single item and all-or-none scoring. Furthermore, reliability of the prospective memory variables was seldom reported (but see Einstein, Smith, McDaniel, \& Shaw, 1997; Maylor, 1996a; and Schmidt et al., 2001, for exceptions), and if it is low, there would be little systematic variance in the variables available to be correlated with other variables, Finally, in most of the studies the constructs were assessed with single variables, which, besides including measurement error, likely represents only a limited aspect of the intended construct and also reflects other influences.

A major goal of the present study was to investigate the construct validity of prospective memory by administering four tasks hypothesized to require prospective memory to a moderately large $(N=330)$ sample of adults who also performed a battery of cognitive tests designed to assess other cognitive abilities. Construct validity was investigated with the four confirmatory factor analysis models described in Salthouse, Atkinson, and Berish (2003), which correspond to progressively more demanding tests of construct validity. Model A simply examines whether variables hypothesized to represent prospective memory are significantly related to a common construct. This model only evaluates convergent validity by determining whether the variables all have significant relations to what they have in common. The next model, Model B, includes variables representing other cognitive constructs, and therefore allows an evaluation of discriminant validity by determining whether the correlations between the target construct (i.e., prospective memory) and other constructs are appreciably less than 1 . Model C extends Model B by allowing individual variables from the target construct to be related to other cognitive abilities if this results in an improved fit of the model to the data. The most rigorous test of construct validity is Model D, in which the target variables are related to all other cognitive ability constructs before examining whether they have significant variance in common.

Salthouse et al. (2003) recently applied this series of models to investigate the construct validity of executive functioning and of cognitive control concepts such as inhibition, updating, and time sharing. The results suggested that the variables included in the analyses did not form distinct constructs, instead appearing to be closely related to the psychometric construct of fluid intelligence $(\mathrm{gF})$. Because this finding was surprising, a secondary goal of the present study was to determine whether these results would be replicated with a different combination of variables used to assess executive functioning.

The methodological concerns discussed above are also relevant when attempting to make comparisons across different types of prospective memory (such as those with different primary task demands or target salience), across different hypothesized components of prospective memory (such as forming, remembering, or executing the intention), or between prospective memory and either 
retrospective memory or a variety of individual difference characteristics. That is, because few if any single variables exclusively and exhaustively represent a given theoretical construct, comparisons derived from a specific task may not accurately reflect that construct.

One particularly interesting hypothesized relation involving prospective memory is with executive functions. The rationale for this relation has recently been stated in the following manner by Mäntylä (2003, p. 24): “different forms of executive functions, such as planning, interruption, response inhibition, self-initiation, and monitoring, are intimately involved in the performance of prospective memory tasks." Similar arguments concerning the possible linkage between executive functions and prospective memory have been provided by McDaniel, Glitsky, Rubin, Guynn, and Routhieaux (1999) and Vogels et al. (2002).

However, closer examination reveals the weakness of the available evidence for the hypothesized linkage to executive functioning. To illustrate, Mäntylä and Nilsson (1997) found a significant correlation between a measure of prospective memory performance and verbal fluency, which was assumed to represent executive functioning. Although verbal fluency is frequently hypothesized to reflect executive functioning, recent research suggests that it is closely related to vocabulary (representing the number of words one knows) and to speed (representing how quickly one can search through the words in his or her knowledge store), and may not be closely related to other purported measures of executive functioning such that a distinct executive functioning construct can be identified (see Salthouse et al., 2003).

Another relevant study was reported by McDaniel et al. (1999), who compared older adults categorized as high $(N=21)$ or low $(N=20)$ on a composite of five variables postulated to reflect executive functioning (i.e., the Wisconsin Card Sorting Test number of categories, verbal fluency, arithmetic, backward digit span, and mental control). While the individuals with high composite scores performed better than the individuals with low composite scores on an event-based prospective memory task, these individuals also had higher scores on tests of vocabulary, word recall, and the Performance IQ measure from the Wechsler Adult Intelligence Scale. This pattern suggests that the advantage of the high-functioning older adults was relatively general, and not specific to aspects of executive functioning. In the present study, participants completed several tasks hypothesized to assess executive functioning and other cognitive abilities to allow an examination of the relation of prospective memory to each of these constructs.

Prospective memory has also been hypothesized to be related to self-reported memory problems (e.g., Groot et al., 2002; Kinsella et al., 1996; Mäntylä, 2003). Some support for this linkage is available because Kinsella et al. found significant correlations between prospective memory measures and self-reported memory problems from the Memory Functioning Questionnaire (MFQ), but not between MFQ factors and measures of retrospective memory. However, the issue is still open because other researchers have found little relation between measures of prospective memory and reported memory problems (e.g., Dobbs \& Rule, 1987; Groot et al. 2002; Zelinski, Gilewski, \& Anthony-Bergstone, 1990). We investigate this question in the present study by having participants complete a modified version of the MFQ (Gilewski, Zelinski, \& Schaie, 1990; Zelinski et al., 1990), and then examining relations of composite scores obtained from this questionnaire to measures of prospective memory.

Still another potential relation involving prospective memory is with noncognitive factors. For example, Einstein and McDaniel (1996) suggested that personality variables such as compulsiveness and conscientiousness might be related to prospective memory performance. Although Goschke and Kuhl (1996) did report relations between personality style and measures of prospective memory, the evidence for this relation is quite limited. This question was investigated in the present study by having participants complete the NEO Five-Factor Personality Inventory (Costa \& McCrae, 2003), and then examining relations between the five personality dimensions obtained from this instrument and measures of prospective memory performance.

Finally, a major question with respect to aging is whether at least some of the age-related influences on the prospective memory construct are unique and independent of the age-related influences on other constructs (Salthouse \& Ferrer-Caja, 2003). That is, a very large number of cognitive variables (and constructs) have been found to be related to age, and only if it were to be established that the age-related effects on a construct were at least partially independent of the age-related effects on other constructs would the relations necessarily warrant a separate explanation. This question will be investigated with the multiple-ability mediational model portrayed in Figure 1. Note that each of the five cognitive abilities is postulated to be influenced by age, and in turn to influence a prospective memory construct. Of particular interest is the coefficient for the dotted arrow, which represents direct relations of age on the prospective memory construct after considering indirect effects through the other cognitive abilities.

The data for this report were collected as part of a larger project in which participants reported to the laboratory for three sessions and performed an extensive battery of cognitive tasks. Sixteen of the tasks were selected to establish a set of five reference cognitive abilities (i.e., reasoning, spatial visualization, episodic memory, perceptual speed, and vocabulary). However, the reasoning and spatial visualization factors were found to be highly correlated with one another (i.e., $r=.90$ ), which resulted in distorted estimates of their unique relations with other constructs due to multicollinearity. These two factors were therefore combined into a single $\mathrm{gF}$ factor in all subsequent analyses. 


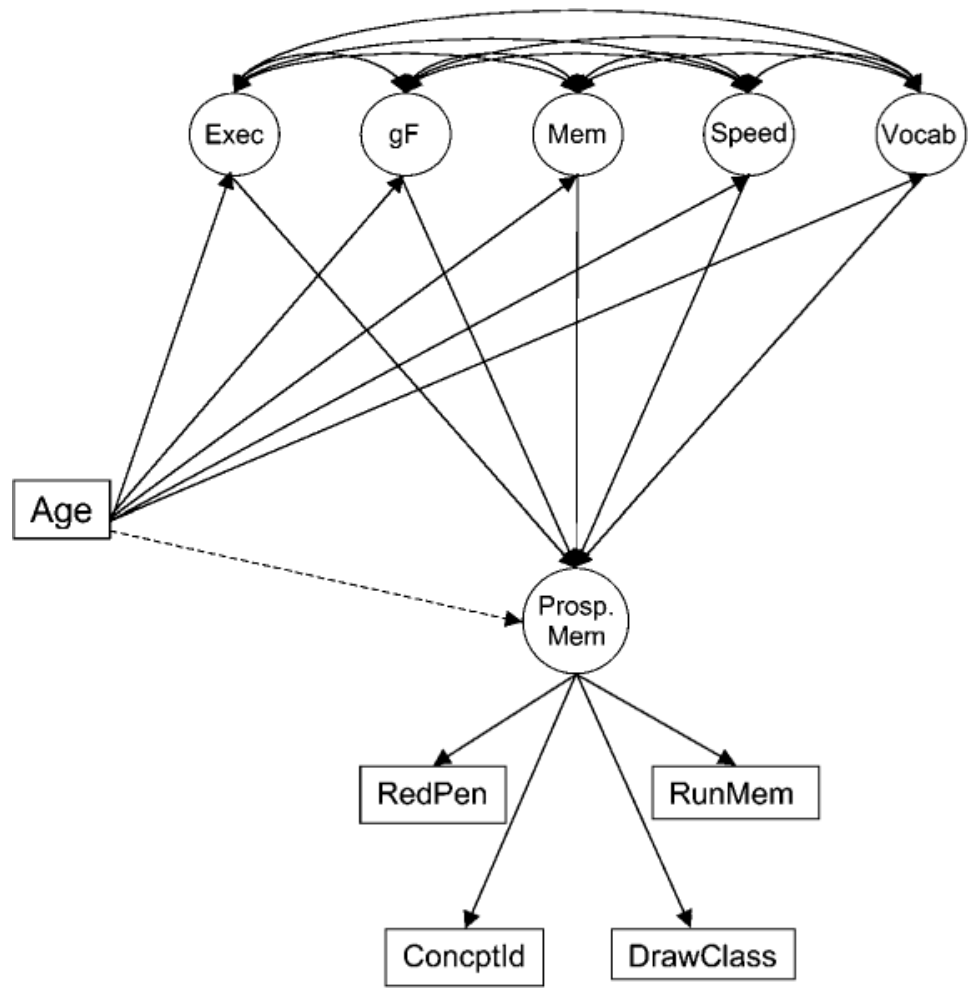

Figure 1. Structural model investigating the role of other cognitive abilities as potential mediators of age-related influences on prospective memory.

To summarize, in the present study, we focused on prospective memory with the following questions: First, is prospective memory a coherent construct as reflected by a common pattern of individual differences across measures from different tasks? Second, how is prospective memory related to executive functioning and to other cognitive abilities? Third, how is prospective memory related to noncognitive factors such as self-rated memory problems and various dimensions of personality? And fourth, to what extent are age-related influences on prospective memory unique and independent of age-related influences on other cognitive abilities?

\section{METHOD}

\section{Participants}

A total of 331 research participants were recruited through newspaper advertisements, posted flyers, and referrals from other participants. The data from one participant were lost, and thus the analyses are based on 330 participants. Each individual reported to the laboratory for three sessions of approximately $2 \mathrm{~h}$ each and also completed several questionnaires at home. Descriptive characteristics of the participants, arbitrarily divided into three age groups, are presented in Table 1.

Inspection of the table reveals that most of the participants rated themselves to be in very good or excellent health, and that on average they had completed over 15 years of formal education. Because the scaled scores from the WAIS III (Wechsler, 1997a) Vocabulary and Digit Symbol tests have means of 10 and standard deviations of 3 , it can be inferred that the sample averaged nearly $2 / 3$ of a stan- dard deviation above the nationally representative normative sample. However, it is important to note that the weak correlation between age and the scaled scores suggests that there was no relation between age and the selectivity of the sample as determined by the Vocabulary and Digit Symbol variables.

Because of the significant correlation of age with self-reported health, the primary analysis based on the model in Figure 1 was repeated after allowing the health variable to be related to age and to all latent constructs. The results from this analysis were very similar to those without the health variable, and thus health status was ignored in all other analyses.

\section{Procedure}

The tests used to assess the reference cognitive abilities were primarily obtained from commercial test batteries. Table 2 briefly describes the tests, their source, and the estimated reliability of the variables.

Executive functioning. Several of the tests described below were obtained from a recent test battery (Delis, Kaplan, \& Kramer, 2001) specifically designed to assess executive functioning.

Sort recognition (Delis et al., 2001). In this task, the participant is shown six cards arranged in two columns and is asked to describe how the cards in each column are alike in some way, and different from the cards in the other column. Each block contains eight displays of six cards each, and the participants' descriptions are assigned 0,1 , or 2 points depending on the accuracy of the description according to criteria provided in the manual. Because 16 points were possible in each of two blocks, the maximum score was 32 .

The task was introduced with two examples involving sets of six cards containing yellow squares or circles with boys' or girls' names on them. In the first trial, the items on the left were circles and those on the right were squares, and in the second trial the items 
Table 1

Means (With Standard Deviations) of Participant Characteristics and Correlations With Age

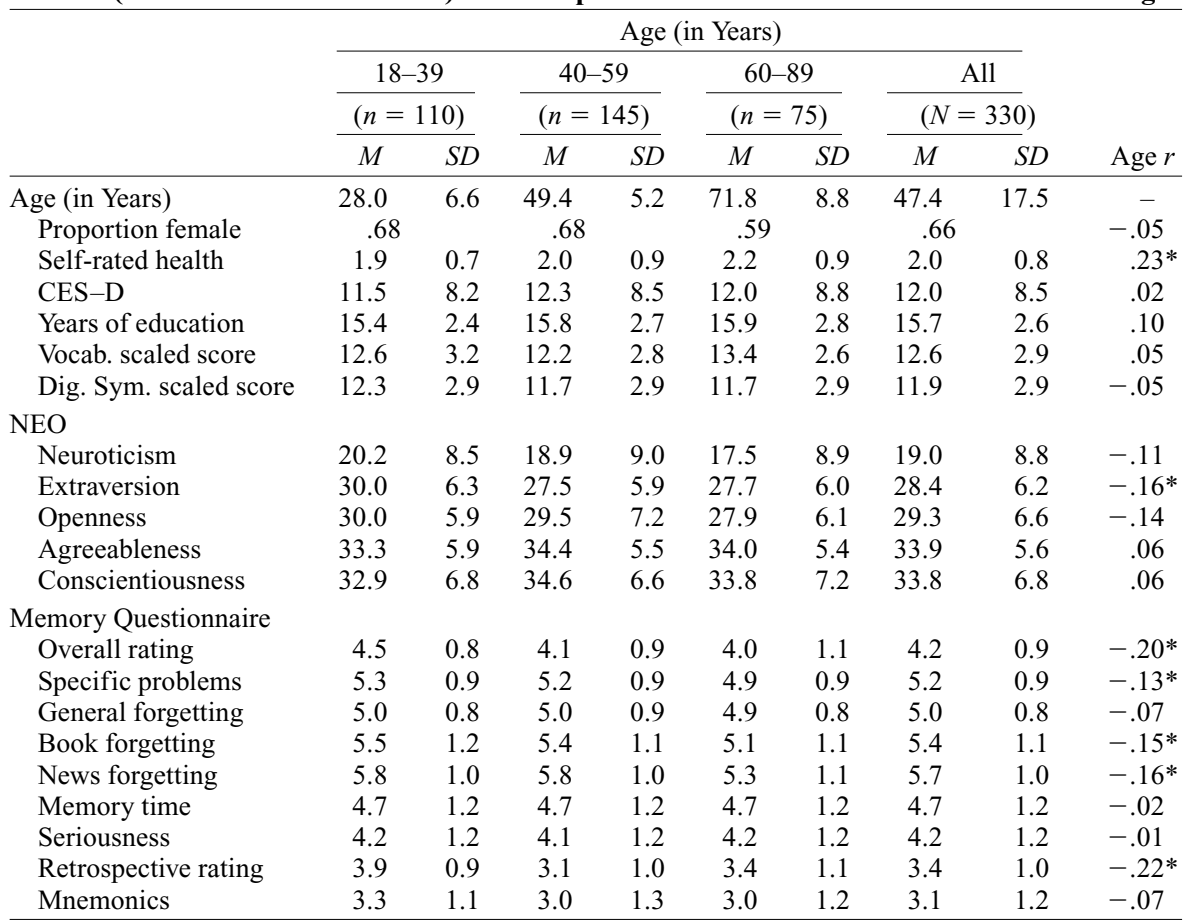

Note-Health was rated on a scale ranging from $1=$ excellent to $5=$ poor. CES-D is the Center for Epidemiological Studies-Depression Scale (Radloff, 1977). Vocabulary Scaled Scores and Digit Symbol Scaled Scores are the age-adjusted scaled scores from the Wechsler Adult Intelligence Scale III (Wechsler, 1997a). The five personality dimensions were assessed with the NEO-FFI (Costa \& McCrae, 2003), and the Memory Questionnaire was a modification of the Memory Functioning Questionnaire (Zelinski et al., 1990). $* p<.01$.

on the left were boys' names and those on the right were girls' names.

After making sure that the participants understood how the cards in the two sample trials were sorted, the first set of eight trials was presented. Each trial was presented for $30 \mathrm{sec}$ and consisted of six cards varying in dimensions such as size of object, category of object, color of card, color of label, number of syllables in object name, upper- or lowercase letters, and so on. A second set of eight trials with different stimuli and dimensions of variation followed immediately after completion of the first set. Reliability of the measure of sort recognition estimated from coefficient alpha was .76.

Multiple-choice proverb interpretation (Delis et al., 2001). In this task, the participant is asked to select the most accurate interpretation of a proverb from a set of four alternatives. Responses are scored according to criteria in the manual, with 4 points assigned for a correct abstract response, 2 points for a correct concrete response, and 0 points for an incorrect phonemically related response or an incorrect unrelated response. Seven new proverbs were added to the original set of eight in an attempt to increase sensitivity, and thus the maximum score in this test was 60 . The coefficient alpha estimate of reliability based on all 15 items was .66.

Verbal fluency (Delis et al., 2001). The verbal fluency test consisted of three conditions in which the participant spoke words as rapidly as possible which were recorded by the examiner. In the letter fluency condition, participants were instructed to name words beginning with a specified letter, but were instructed not to use names of persons, places, numbers, or words that differed only with respect to the ending. On successive trials, each of which lasted
$60 \mathrm{sec}$, the target letters were F, A, and S. In the category condition, $60 \mathrm{sec}$ were allowed for the participant to name words that fell within a specified category, with the first category consisting of animals and the second consisting of boys' names. In the category fluency condition, $60 \mathrm{sec}$ were allowed for the participant to name words from two alternating categories, starting with fruits and then furniture, and continuing to alternate between these two categories with each successive response. Estimated reliability in the letter fluency condition was .86 , and in the category fluency condition it was .67. Because there was only one trial in the alternating fluency condition, reliability could not be computed from the data in this study, but a test-retest reliability of .52 is reported in the manual for this measure.

Connections. This task was described in Salthouse et al. (2000) and was designed as an improvement of the trail making test in that the length of the path connecting successive items in the different conditions was controlled, the single and alternating trials were counterbalanced, and the single conditions included both alphabetic and numeric versions. The materials consist of pages with 49 circles containing letters or numbers that were to be connected as quickly as possible by drawing lines according to numerical, alphabetical, or alternating numerical and alphabetical sequence.

After the task was explained and participants completed two sample trials, eight pages were administered with a time limit of $20 \mathrm{sec}$ each. The pages were presented in the order numbers, numbers-letters, letters-numbers, letters, letters, letters-numbers, numbers-letters, and numbers. On each page, the number of incorrect responses was subtracted from the number of correct responses, and this number was 
Table 2

Description of Tests Used to Assess Cognitive Abilities

\begin{tabular}{|c|c|c|c|}
\hline Test & Description & Source & Reliability \\
\hline $\begin{array}{l}\text { Raven's } \\
\text { progressive } \\
\text { matrices }\end{array}$ & $\begin{array}{l}\text { Select best completion of } \\
\text { missing cell in a matrix }\end{array}$ & Raven (1962) & .85 \\
\hline Letter sets & $\begin{array}{l}\text { Determine which set of } \\
\text { letters does not belong with } \\
\text { the others }\end{array}$ & $\begin{array}{l}\text { Ekstrom, French, Harman, \& Dermen } \\
\text { (1976) }\end{array}$ & .80 \\
\hline Analytical reasoning & $\begin{array}{l}\text { Select which alternative best } \\
\text { satisfies a set of constraints }\end{array}$ & GRE (1994) & .94 \\
\hline Spatial relations & $\begin{array}{l}\text { Determine which 3-D object } \\
\text { corresponds to an unfolded } \\
\text { 2-D diagram }\end{array}$ & Bennett, Seashore, \& Wesman (1997) & .81 \\
\hline Paper folding & $\begin{array}{l}\text { Determine which pattern of } \\
\text { holes corresponds to a } \\
\text { sequence of paper folds and hole punch }\end{array}$ & Ekstrom et al. (1976) & .84 \\
\hline Form boards & $\begin{array}{l}\text { Determine which combination } \\
\text { of shapes are needed to fill } \\
\text { a larger shape }\end{array}$ & Ekstrom et al. (1976) & .90 \\
\hline Logical memory & $\begin{array}{l}\text { Immediate recall of details } \\
\text { from a short text }\end{array}$ & Wechsler (1997b) & .72 \\
\hline Free recall & $\begin{array}{l}\text { Immediate recall of a list } \\
\text { of unrelated words on } \\
4 \text { successive trials }\end{array}$ & Wechsler (1997b) & .79 \\
\hline Paired associates & $\begin{array}{l}\text { Recall of the response term } \\
\text { when presented with the } \\
\text { stimulus term of six unrelated } \\
\text { word pairs }\end{array}$ & Salthouse, Fristoe, \& Rhee (1996) & .71 \\
\hline Digit symbol & $\begin{array}{l}\text { Write symbols below digits } \\
\text { according to a code table }\end{array}$ & Wechsler (1997a) & .84 \\
\hline Letter comparison & $\begin{array}{l}\text { Classify sets of letters as } \\
\text { same or different as quickly } \\
\text { as possible }\end{array}$ & Salthouse \& Babcock (1991) & .71 \\
\hline Pattern comparison & $\begin{array}{l}\text { Classify sets of line patterns } \\
\text { as same or different as quickly } \\
\text { as possible }\end{array}$ & Salthouse \& Babcock (1991) & .73 \\
\hline WAIS vocabulary & Provide definitions of words & Wechsler (1997a) & .85 \\
\hline $\begin{array}{l}\text { Woodcock-Johnson } \\
\text { picture vocabulary }\end{array}$ & Name the pictured objects & Woodcock \& Johnson (1990) & .81 \\
\hline Synonym vocabulary & $\begin{array}{l}\text { Select the best synonym } \\
\text { of the target word }\end{array}$ & Salthouse (1993) & .82 \\
\hline Antonym vocabulary & $\begin{array}{l}\text { Select the best antonym } \\
\text { of the target word }\end{array}$ & Salthouse (1993) & .83 \\
\hline
\end{tabular}

Note-Reliabilities are estimated from coefficient alpha, with the exception of the digit symbol task, for which the value is the test-retest coefficient reported in the manual.

divided into 20 to obtain a score in seconds per item. These scores were then averaged across the four pages with items of the same type (i.e., all numbers or all letters) to yield a "same" score, and averaged across the four pages with alternating items (i.e., numbers-letters, letters-numbers) to yield an "alternating" score. The estimated reliability of the difference in time between same and alternating sequences was .66.

Prospective memory. All participants performed four tasks designed to assess prospective memory. At the completion of each task, the participants were asked whether they remembered if they were supposed to do something special in addition to the primary (i.e., ongoing) task, and if so, to describe what it was. If the participant successfully described the response (i.e., the phrase to be spoken or the key to be pressed), he or she was considered to have remembered the task instructions. Many of the participants also described the target stimulus in their answers, but unfortunately this information was not recorded.
Red pencil. This task was loosely based on a task described by Dobbs and Rule (1987) in which participants were instructed to request a pencil at a particular time in the session. In our version of the task, participants were instructed to repeat the words "red pencil" whenever those words were said by an examiner. Participants were told that this phrase could occur at any point during the three experimental sessions, and that they would not receive any further reminders of this instruction. The initial instructions for this task were repeated and clarified as necessary until the participant repeated the phrase.

Two "red pencil" events were included in each session in the context of the instructions for one of the cognitive tasks. To illustrate, in one of the tasks the examiner stated, "You may use either the black or red pencil in this task." The three testing sessions were scheduled at each participant's convenience, and thus the time interval between successive red pencil "trials" varied across participants from one day to several weeks or more. ${ }^{1}$ The question about 
memory for the task instructions was asked only at the end of the third session. Performance in this task was assessed as the proportion of "red pencil" events in which the participant repeated the words "red pencil."

Drawing classification. This task, which was performed in the second session, was based on the pictures task described by Vogels et al. (2002). Participants were instructed to classify black and white line drawings as representing living or nonliving objects by pressing the " 1 " key for living and the " 0 " key for nonliving. In addition to this living/nonliving judgment, they were instructed to make a prospective memory response by pressing the "2" key whenever they saw a drawing representing a designated target object. The task was organized into 15 blocks of between 7 and 12 drawings each. At the beginning of each block, a target word representing the name of an object was displayed, and the participant was instructed to press the " 2 " key whenever a drawing of that object was presented in the next set of drawings. The target drawing appeared in 9 of the 15 blocks, and it always occurred as the third-to-last picture in a given block. The measure of prospective memory performance in this task was the proportion of hits (i.e., press of the "2" key to a target object) minus the proportion of false alarms (i.e., press of the " 2 " key to a nontarget object).

Concept identification. In this task, which was performed in the third session, the participants were instructed to assign multidimensional stimuli to one of four categories while simultaneously remembering to make a different response to a designated target stimulus. The stimuli consisted of shapes (i.e., circles, squares, crosses, and stars) that varied in color (i.e., red, blue, green, and orange) and number (i.e., one, two, three, and four). The procedure resembled the Wisconsin Card Sorting Test in that the dimension for a correct categorization changed throughout the task. Eight successive trials were presented with each sorting dimension, in this order: color, shape, number, shape, color, and number. Four reference stimuli (i.e., one red star, two green circles, three blue squares, and four orange crosses) were always displayed at the top of the screen, and participants assigned stimuli to one of the categories by pressing a key from 1 to 4 according to the position of the reference stimulus. Feedback in the form of the words "correct" or "incorrect" was displayed after each response.

The prospective memory target, a display of four green stars, appeared on the eighth trial of the first, second, fourth, and sixth sets of trials. Participants were instructed to press the " 0 " key whenever the prospective memory target occurred and to ignore the categorization task for that trial. The measure of prospective memory performance in this task was the proportion of hits (i.e., press of the " 0 " key to a display of four green stars) minus the proportion of false alarms (i.e., press of the "0" key to any other display).

Running memory. The running memory task, performed in the third session, was based on the event-based prospective memory task described in Park et al. (1997; see also Kidder et al., 1997). Participants were presented with words in one of six different backgrounds and were instructed to attempt to keep track of the most recently presented three words while also making a specific response whenever the designated background was presented. A block consisted of 72 trials, and there were six probes requesting recall of the last three words in each block. The two blocks involved different words and backgrounds. At each probe, the examiner wrote the words spoken by the participant, and then restarted the sequence of word-background displays.

At the beginning of each block, a prospective memory target background was displayed, and participants were instructed to press the " 0 " key whenever that background appeared and to press the " 1 " key for all other backgrounds. The target background appeared 12 times in each block of 72 trials. Different backgrounds and words were used in each block of trials. The measure of prospective memory performance in this task was the proportion of hits (i.e., press of the " 0 " key to the target background) minus the proportion of false alarms (i.e., press of the " 0 " key to a nontarget background).

To summarize, each participant performed four prospective memory tasks that involved different primary or ongoing activities, different numbers of targets (i.e., from 4 to 24), different relations of the target stimuli to the primary task (i.e., some were incidental to the primary activity as in the red pencil and running memory tasks, and in others the target stimulus was relevant to the primary activity as in the drawing classification and concept identification tasks); two involved the same prospective memory target on all trials (i.e., red pencil and concept identification), two involved a variety of different prospective memory targets (i.e., running memory and drawing classification), one (red pencil) involved a vocal response, and three (drawing classification, concept identification, and running memory) involved a keypress response. Although the prospective memory tasks varied in a number of dimensions, it should be noted that the range was still somewhat restricted because the tasks were performed in a laboratory context, all but one occurred over a relatively short time period, and in none of the tasks did a failure have much consequence in one's life.

\section{RESULTS}

We have organized the description of the results into six sections. ${ }^{2}$ The first section summarizes performance on the variables used to establish the reference cognitive abilities. The second section describes performance on the primary activity in each prospective memory task, and the third considers prospective memory performance and its relations to age. The fourth section reports the correlational analyses designed to investigate the construct validity of executive functioning and of prospective memory, and the fifth section describes the results relevant to the uniqueness of age-related influences on prospective memory. The final section describes correlations between prospective memory and dimensions of personality, and self-reports of memory functioning.

\section{Reference Cognitive Variables}

Means, standard deviations, and age correlations for the variables that served to identify the reference cognitive abilities are contained in Table 3. As expected, nearly all of the variables except for those assessing vocabulary had significant negative correlations with age, indicating that increased age was associated with lower performance. The letter fluency variable is an exception, but it is worth noting that this correlation was also significantly negative (i.e., - .19) after statistically controlling for the variance in a composite measure of vocabulary formed by averaging the $z$ scores for the four vocabulary variables.

\section{Primary Activity Performance}

In the red pencil task, the participant was to repeat the phrase "red pencil" whenever it was said by the examiner. Because the prospective "events" occurred during the reading of instructions for other tasks, there was no primary activity associated with this task.

In the running memory task, the participant was to repeat the last three words in the sequence whenever the word RECALL appeared in the display. The proportion of 
Table 3

Means, Standard Deviations, and Age Correlations of Reference Cognitive Variables

\begin{tabular}{lrrr}
\hline & $M$ & $S D$ & Age $r$ \\
\hline gF & & & \\
Raven's progressive matrices & 7.8 & 3.3 & $-.60^{*}$ \\
Letter sets & 11.1 & 3.0 & $-.41^{*}$ \\
Analytical reasoning & 3.8 & 2.7 & $-.52^{*}$ \\
Spatial relations & 9.1 & 5.0 & $-.39^{*}$ \\
Paper folding & 6.9 & 2.9 & $-.42^{*}$ \\
Form boards & 6.7 & 4.3 & $-.44^{*}$ \\
Memory & & & \\
Logical memory & 46.6 & 10.1 & $-.25^{*}$ \\
Free recall & 37.2 & 6.5 & $-.43^{*}$ \\
Paired associates & 3.2 & 1.9 & $-.33^{*}$ \\
Speed & & & \\
Digit symbol & 77.6 & 18.4 & $-.62^{*}$ \\
Letter comparison & 11.2 & 2.6 & $-.41^{*}$ \\
Pattern comparison & 17.6 & 3.6 & $-.52^{*}$ \\
Vocabulary & & & \\
WAIS vocabulary & 51.1 & 10.1 & .11 \\
Woodcock-Johnson picture vocabulary & 18.8 & 5.4 & $.31^{*}$ \\
Synonym vocabulary & 7.0 & 2.8 & $.32^{*}$ \\
Antonym vocabulary & 6.5 & 3.1 & $.31^{*}$ \\
Executive Functioning & & & \\
Sort recognition & 12.6 & 5.9 & $-.45^{*}$ \\
Letter fluency & 14.1 & 4.1 & -.06 \\
Category fluency & 20.5 & 4.6 & $-.21^{*}$ \\
Alternating fluency & 15.0 & 3.0 & $-.20^{*}$ \\
Connections difference & 0.8 & 0.6 & $.23^{*}$ \\
Proverb interpretation & 58.0 & 4.2 & $-.21^{*}$ \\
\hline & & & \\
\hline$<.01$ ran & & &
\end{tabular}

$* p<.01$.

Table 4

Summary Statistics for Prospective Memory Variables

\begin{tabular}{lcccc}
\hline \multicolumn{1}{c}{ Variable } & $M$ & $S D$ & Est. Rel. & Age $r$ \\
\hline Red pencil & .74 & .37 & .93 & $-.40^{*}$ \\
Running memory & .79 & .22 & .61 & $-.32^{*}$ \\
Drawing classification & .91 & .17 & .81 & $-.28^{*}$ \\
Concept identification & .72 & .37 & .87 & $-.22^{*}$ \\
\hline
\end{tabular}

Note-Est. Rel., estimated reliability obtained from coefficient alpha; Age $r$, Pearson product-moment correlation of the variable with age. $* p<.01$.

words recalled correctly decreased as a function of sequence position because the means were $.90, .80$, and .61 , respectively, for words one, two, and three back in the sequence. The magnitude of the age correlation also varied across sequence positions because the correlation was -.20 for recall of words one back in the sequence, but -.27 for words two back, and -.34 for words three back in the sequence. All three of these correlations were significantly $(p<.01)$ different from zero, as were the semipartial correlations for two-back words controlling for memory of one-back words (i.e., - .16), and for threeback words controlling for memory of two-back words (i.e., -.18 ).

Participants in the drawing classification task were to classify drawings as representing living or nonliving objects. Accuracy averaged $95 \%$, and the correlation with age was not significantly different from zero (i.e., $r=$
$-.13)$. Classification accuracy was not perfect, probably in part because the portrayals of a few of the stimulus items were ambiguous with respect to the living/nonliving classification, such as the drawings of cherries, a feather, and mistletoe.

In the concept identification task, the participant had to determine the rules used to assign stimuli to categories, and then use those rules to sort new stimuli. The average accuracy was $58 \%$, and accuracy was not significantly correlated with age (i.e., $r=-.08$ ). The level of accuracy was not very high, possibly because the participants had to discover the rule for classifying stimuli, and there was an unannounced change in the rule every eight trials. In addition, on four trials the feedback was based on the prospective memory component (i.e., target or nontarget response) rather than on the current classification rule in the concept identification task, and this could have confused some participants.

\section{Prospective Memory Performance}

Table 4 contains the means, estimates of reliability, and age correlations for the prospective memory variables. ${ }^{3}$ The red pencil score is the proportion of events with an appropriate response (i.e., saying "red pencil"), and scores in the running memory, drawing classification, and concept identification tasks are hit rates minus false alarm rates. Because the false alarm rates in these tasks averaged only $.01, .00$, and .01 , respectively, the scores are primarily determined by the hit rates. Coefficient alpha estimates of reliability were obtained by treating individual trials as items. With the exception of the running memory variable, which had an estimated reliability of .61, the reliabilities were all greater than .80 .

The age trends by decade for the four prospective memory variables are illustrated in Figure 2. The age relations appear nonlinear, with little decline in any of the variables until after about age 50 . These trends were investigated in multiple regression analyses in which agesquared (quadratic) terms were entered after the (linear) age terms. The nonlinear $\left(\mathrm{age}^{2}\right)$ effects were significant $(p<.01)$ for each variable as the standardized coefficients were .042 for drawing classification, .064 for concept identification, .067 for running memory, and .033 for red pencil. These results suggest that the patterns may be different for adults under and over about age 50, a possibility that was investigated in later analyses.

Before further examining age relations on the prospective memory variables, the effects of two factors need to be considered: level of performance in the primary task and retrospective memory of task instructions. The former is important because there could be a tradeoff between prospective memory performance and performance in the primary task, and the latter is important because performance on the prospective task could be low if one forgets what one is supposed to do.

We investigated the role of primary task performance by computing correlations between prospective memory performance and performance on the primary tasks. There 


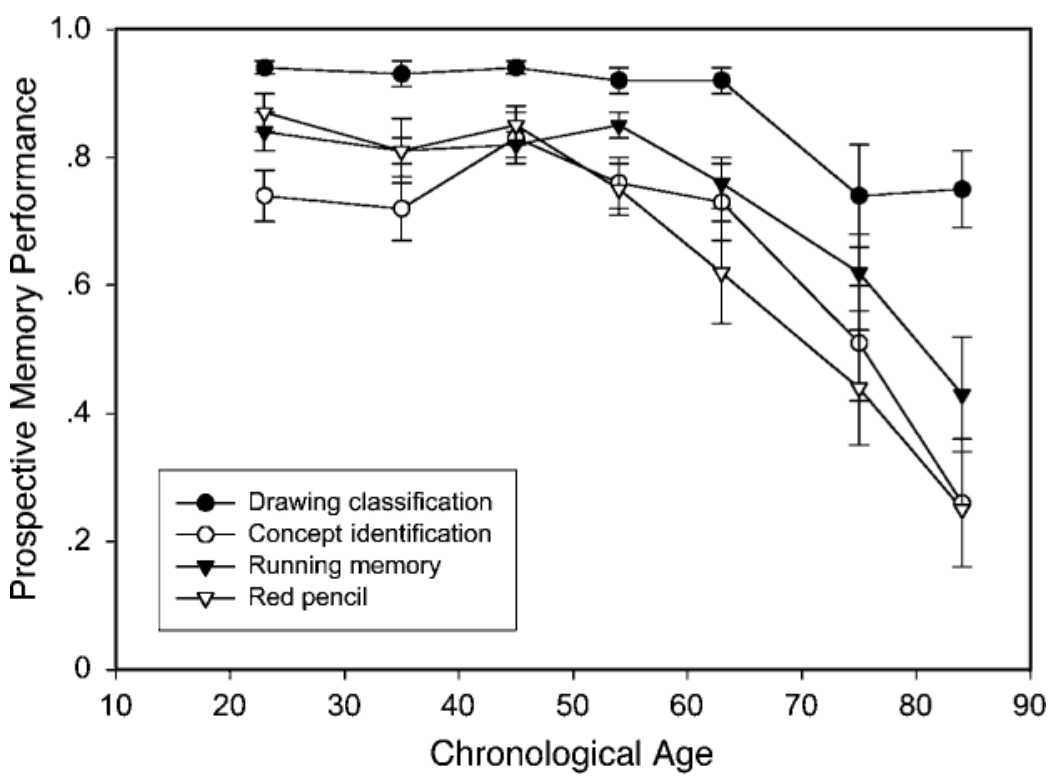

Figure 2. Means (and standard errors) by decade of performance on the four prospective memory variables. The numbers of individuals contributing to the scores for each decade were $67,43,71,74,34,24$, and 17 , respectively, from the youngest (18-29) to the oldest $(80-89)$ groups.

was no primary activity in the red pencil task, and thus it was not possible to compute a correlation in this task. The correlations for the other tasks were $.33(p<.01)$ in the running memory task, $.26(p<.01)$ in the drawing classification task, and .05 (n.s.) in the concept identification task. Two of the correlations are significantly positive, indicating that high accuracy in the primary task was associated with high accuracy in the prospective memory task. Because this pattern is inconsistent with a tradeoff in which prospective memory performance is lower when the primary task performance is higher, primary task performance was ignored in subsequent analyses.

Table 5 contains means, standard deviations, and pointbiserial age correlations for the binary variables of whether the participant remembered the prospective memory instruction in each task. Data are missing in a number of cases because the examiner forgot to ask the question at the appropriate time (reflecting a prospective memory failure on the part of the examiner). Inspection of the

Table 5

Summary Statistics for Measures of Memory for Task Instructions

\begin{tabular}{lcccc}
\hline \multicolumn{1}{c}{ Task Instruction } & $N$ & $M$ & $S D$ & Age Corr. \\
\hline Red pencil & 223 & .84 & .37 & $-.39^{*}$ \\
Running memory & 274 & .96 & .20 & $-.26^{*}$ \\
Drawing classification & 273 & .97 & .17 & $-.22^{*}$ \\
Concept identification & 278 & .93 & .26 & $-.31^{*}$ \\
\hline
\end{tabular}

Note-Age Corr., point-biserial correlation of the Yes/No task instruction memory variable with age. $* p<.01$. table reveals that accuracy of remembering the prospective memory instructions was very high except in the red pencil task. Nevertheless, increased age was associated with significantly lower accuracy in each task.

Three methods were used to analyze age relations on the prospective memory variables after controlling for the influence of retrospective memory for the prospective memory task instructions. One method involved the use of statistical control (by means of hierarchical regression) of the variable representing memory for the task instruction when analyzing the relation of the prospective memory variable to age. Another method involved restricting the analyses to participants who correctly reported the task instructions, and the third method consisted of restricting the analyses to participants who had at least one correct prospective memory response.

As one might expect, posttask memory for the prospective memory task instructions was higher when participants had at least one correct prospective memory response. That is, the proportion of individuals who remembered the red pencil task instructions was .63 when no prospective memory responses were correct, but it was .93 when they had at least one correct prospective memory response. Corresponding values in the running memory, drawing classification, and concept identification tasks were 0 and $.97, .25$ and .99 , and .67 and .97 , respectively.

Table 6 contains the results of the analyses in which retrospective memory of the task instructions was statistically controlled. It can be seen that the age correlations are all reduced after controlling for memory of task in- 
Table 6

Relations of Prospective Memory Variables to Age Before and After Controlling for Retrospective Memory of Task Instructions

\begin{tabular}{lcccc}
\hline \multicolumn{1}{c}{ Task Instruction } & $\begin{array}{c}\text { Simple } \\
\text { Correlation }\end{array}$ & $\begin{array}{c}\text { After Control } \\
\text { Retrospective Memory }\end{array}$ & $\begin{array}{c}\text { Only Participants } \\
\text { With Correct } \\
\text { Retrospective Memory }\end{array}$ & $\begin{array}{c}\text { Only Participants } \\
\text { With }>0\end{array}$ \\
\hline Red pencil & $-.40^{*}$ & $-.13^{*}$ & $-.20^{*}$ & $-.20^{*}$ \\
Running memory & $-.32^{*}$ & $-.23^{*}$ & $-.27^{*}$ & $-.27^{*}$ \\
Drawing classification & $-.28^{*}$ & $-.20^{*}$ & $-.25^{*}$ & $-.25^{*}$ \\
Concept identification & $-.21^{*}$ & -.12 & -.11 & -.11 \\
\hline
\end{tabular}

Note-The first column of data contains the Pearson product-moment correlations of the variable with age. The second column contains the semipartial correlation of the variable with age after controlling for the variance in the task instruction memory measure. The third and fourth columns contain the Pearson productmoment correlations only for participants who remembered the task instructions (3rd column) or who were correct on at least one prospective memory event (4th column). $\quad * p<.01$.

structions, which suggests that at least some of the agerelated effects on these measures of prospective memory are attributable to retrospective memory failure. However, in three of the four variables the age relations were still significant even after these controls, and thus apparently not all of the age-related effects on the prospec-

Table 7

Statistics for Construct Validity Models of Executive Functioning (EF) Construct

\begin{tabular}{|c|c|c|c|c|c|}
\hline & & \multicolumn{4}{|c|}{ Model } \\
\hline & & A & $\mathrm{B}$ & $\mathrm{C}$ & $\mathrm{D}$ \\
\hline \multicolumn{6}{|l|}{ Fit statistics } \\
\hline$\chi^{2}$ & & 40 & 643 & 481 & 439 \\
\hline$d f$ & & 9 & 199 & 194 & 179 \\
\hline CFI & & .99 & .98 & .99 & .99 \\
\hline RMSEA & & .10 & .08 & .07 & .07 \\
\hline \multicolumn{6}{|c|}{ Loadings on EF construct } \\
\hline $\mathrm{EF} \rightarrow$ Sort & & $.45^{*}$ & $.67 *$ & .06 & -.05 \\
\hline $\mathrm{EF} \rightarrow \mathrm{Con} 1$ & & $-.32 *$ & $-.47 *$ & -.07 & .02 \\
\hline $\mathrm{EF} \rightarrow$ Prov & & $.31 *$ & $.43^{*}$ & .09 & .03 \\
\hline $\mathrm{EF} \rightarrow$ LetF & & $.67 *$ & $.59 *$ & $.59^{*}$ & -.43 \\
\hline $\mathrm{EF} \rightarrow \mathrm{CatF}$ & & $.89 *$ & $.69^{*}$ & $.90 *$ & -.69 \\
\hline $\mathrm{EF} \rightarrow \mathrm{AltF}$ & & $.60 *$ & $.50^{*}$ & $.61 *$ & -.42 \\
\hline \multicolumn{6}{|c|}{ Correlations with other constructs } \\
\hline $\mathrm{EF} \leftrightarrow \mathrm{gF}$ & & & $.75^{*}$ & $.46^{*}$ & \\
\hline $\mathrm{EF} \leftrightarrow \mathrm{Men}$ & & & $.78^{*}$ & $.56^{*}$ & \\
\hline $\mathrm{EF} \leftrightarrow$ Spee & & & $.77^{*}$ & $.60 *$ & \\
\hline $\mathrm{EF} \leftrightarrow$ Voca & & & $.47^{*}$ & $.33^{*}$ & \\
\hline \multicolumn{6}{|c|}{ Loadings on other constructs } \\
\hline Model C & $\mathrm{gF}$ & Mem & \multicolumn{2}{|l|}{ Spd } & Voc \\
\hline SortRecog & $.55^{*}$ & $.19^{*}$ & \multirow{6}{*}{\multicolumn{2}{|c|}{$-.39^{*}$}} & \\
\hline ConnDiff & & & & & \\
\hline Proverbs & & $.36^{*}$ & & & \\
\hline LetFlu & & & & & $.23 *$ \\
\hline CatFlu & & & & & \\
\hline AltFlu & & & & & \\
\hline \multicolumn{6}{|l|}{ Model D } \\
\hline SortRecog & $.56^{*}$ & $.22 *$ & \multicolumn{2}{|c|}{.02} & -.01 \\
\hline ConnDiff & $-.26^{*}$ & -.11 & \multicolumn{2}{|c|}{-.14} & -.05 \\
\hline Proverbs & -.12 & .18 & \multicolumn{2}{|c|}{$.32 *$} & $.21 *$ \\
\hline LetFlu & -.09 & .05 & \multicolumn{2}{|c|}{$.37 *$} & $.39 *$ \\
\hline CatFlu & -.12 & $.18^{*}$ & \multicolumn{2}{|c|}{$.48 *$} & $.23 *$ \\
\hline AltFlu & -.19 & $.32 *$ & \multicolumn{2}{|c|}{$.33 *$} & .01 \\
\hline
\end{tabular}

Note-CFI, comparative fit index; RMSEA, root mean square error of approximation. $* p<.01$. tive memory variables can be attributed to a failure to remember task instructions.

\section{Correlational Analyses of Construct Validity}

The first set of correlational analyses investigated the construct validity of an executive functioning construct with the four analytical models described earlier. Fit statistics and parameter estimates for these models are presented in Table 7. The values for the fit statistics in the top four rows indicated that the models all had reasonable fits to the data. The fits undoubtedly could have been improved by postulating more complex models in which the reference variables were related to more than one ability construct or residuals were allowed to be correlated, but the fit to this relatively simple structure is sufficient to allow meaningful investigations of construct validity.

As can be seen in the first column of Table 7, the variables were all significantly related to the variance they had in common, and thus the results from Model A suggest that executive functioning assessed by these six variables may have convergent validity. The Model B results in the second column are also consistent with the existence of a distinct executive functioning construct because the relations of the construct to the hypothesized indicator variables were all significantly different from zero, providing evidence of convergent validity, and the correlations between that construct and constructs representing other cognitive abilities were only moderate in magnitude, providing evidence of discriminant validity. However, results from the two more stringent models in which the target variables were allowed to be related to other ability constructs that were correlated with the executive functioning construct (Model C), or were related to all of the other ability constructs before examining whether they were significantly related to one another (Model D), were inconsistent with the hypothesized executive functioning construct having construct validity. In Model C, three of the variables no longer had significant variance in common with the other variables that were hypothesized to represent executive functioning when they were allowed to be related to other cognitive 
Table 8

Statistics for Construct Validity Models of Prospective Memory (ProsMem) Construct

\begin{tabular}{|c|c|c|c|c|c|}
\hline & & \multicolumn{4}{|c|}{ Model } \\
\hline & & A & $\mathrm{B}$ & $\mathrm{C}$ & $\mathrm{D}$ \\
\hline \multicolumn{6}{|l|}{ Fit statistics } \\
\hline$\chi^{2}$ & & 0.52 & 761 & 744 & 728 \\
\hline$d f$ & & 2 & 284 & 282 & 269 \\
\hline CFI & & 1.00 & .98 & .98 & .98 \\
\hline RMSEA & & .00 & .07 & .07 & .07 \\
\hline \multicolumn{6}{|c|}{ Loadings on prospective memory construct } \\
\hline ProsMem $\rightarrow$ Red I & & $.47 *$ & $.55^{*}$ & $.36^{*}$ & $.20 *$ \\
\hline ProsMem $\rightarrow$ Runn & Memory & $.56^{*}$ & $.59 *$ & $.63^{*}$ & $.29 *$ \\
\hline ProsMem $\rightarrow$ Draw & g Classif. & $.57 *$ & $.52 *$ & $.55^{*}$ & $.46^{*}$ \\
\hline ProsMem $\rightarrow$ Conc & t Ident. & $.51^{*}$ & $.44^{*}$ & $.46^{*}$ & $.39 *$ \\
\hline \multicolumn{6}{|c|}{ Correlations with other constructs } \\
\hline ProsMem $\leftrightarrow$ Exec & unct. & & $.74 *$ & $.70 *$ & \\
\hline ProsMem $\leftrightarrow \mathrm{gF}$ & & & $.69^{*}$ & $.61^{*}$ & \\
\hline ProsMem $\leftrightarrow$ Mem & & & $.74 *$ & $.73 *$ & \\
\hline ProsMem $\leftrightarrow$ Spee & & & $.63 *$ & $.57^{*}$ & \\
\hline ProsMem $\leftrightarrow$ Voca & lary & & $.22 *$ & $.28^{*}$ & \\
\hline \multicolumn{6}{|c|}{ Loadings on other constructs } \\
\hline Model C & Exec & $\mathrm{gF}$ & Mem & Spd & Voc \\
\hline $\begin{array}{l}\text { Red pencil } \\
\text { Running memory } \\
\text { Drawing classif. } \\
\text { Concept. ident. }\end{array}$ & & $.28 *$ & & & $-.19 *$ \\
\hline \multicolumn{6}{|l|}{ Model D } \\
\hline Red pencil & $.52 *$ & $.28^{*}$ & .03 & -.20 & $-.32 *$ \\
\hline Running memory & .14 & .09 & $.30 *$ & .04 & -.04 \\
\hline Drawing classif. & -.18 & .19 & $.42 *$ & .03 & .02 \\
\hline Concept ident. & .38 & .05 & .15 & -.18 & -.16 \\
\hline
\end{tabular}

Note-CFI, comparative fit index; RMSEA, root mean square error of approximation. $* p<.01$.

ability constructs. Indeed, only the fluency variables appeared to exhibit convergent validity in Model C. The Model D results indicated that there was no significant residual variance common among the variables after their relations to other cognitive ability constructs were taken into consideration. Furthermore, the entries in the bottom of Table 7 suggest that the variables primarily reflect mixtures of other cognitive abilities. For example, the sort recognition variable is related to the $\mathrm{gF}$ and memory abilities, and the fluency variables are related to speed and either vocabulary or memory abilities.

Our conclusion from the analyses summarized in Table 7 is that the variables used to assess executive functioning in this study do not form a coherent and robust construct. The evidence from the weak models suggested that the construct might have both convergent and discriminant validity, but there was little evidence for either type of validity in the more demanding models in which the target variables were allowed to be related to other constructs.

Table 8 contains the results of the four construct validity models applied to the variables hypothesized to represent prospective memory. Because the variables had significant relations to their hypothesized construct in all four models, there was strong evidence for convergent validity. There was also evidence for discriminant validity because the highest correlations with other cognitive ability constructs in Models B and C were .74, which is considerably less than 1 . In contrast to the situation with executive functioning, therefore, the prospective memory construct in this study appears to satisfy both convergent and discriminant aspects of construct validity.

Because the results in Figure 2 suggested the presence of nonlinear age trends on the prospective memory variables, the Model B analysis including both executive functioning and prospective memory variables was repeated with the sample divided into participants between 18 and 49 years of age and between 50 and 89 years of age. We then investigated a series of multiple-group models in which each unstandardized regression coefficient or covariance was constrained to be equal across the two groups to determine which constraints resulted in a significant decrease in fit. A significant reduction in fit would indicate that, in the context of the variables and interrelations included in the model, the parameter differed significantly across the two groups. The coefficients for the two groups are presented in the second and third columns of Table 9, and coefficients that differed significantly $(p<.01)$ between groups are indicated with a " $<$ " sign. Inspection of the table reveals that the pattern was very similar in the two age groups, with significant differences in only a few coefficients. The most interesting differences in the present context were that three of the variables had stronger loadings on the prospective memory construct in the older group than in the younger group. This suggests that prospective memory is a stronger and more coherent construct among adults over the age of 50 than among adults under the age of 50 .

The weaker relations among the prospective memory variables in adults under the age of 50, and the absence of relations of the variables to age in that age group may both be reflections of a functional measurement ceiling. In support of this interpretation are the findings that the variance in the under-50 group was much smaller than that in the 50-and-over group for all four variables. That is, the ratios of the variance in the younger group to the variance in the older group were $.47, .52, .21$, and .62 for the red pencil, running memory, drawing classification, and concept identification tasks, respectively. Lower variance is often associated with a restriction of range that tends to reduce the magnitude of correlations involving the affected variable. Furthermore, the estimated reliabilities were lower in the under-50 group than in the 50 -and-over group for all of the prospective memory variables, and the differences were particularly pronounced for the drawing classification (i.e., .52 vs. .88) and running memory (i.e., .33 vs. .72) variables.

\section{Mediation of Age-Related Effects}

We next investigated statistical mediation effects of age on prospective memory with the structural model portrayed in Figure 1. The initial analysis revealed that the standardized coefficient for the direct relation from 
Table 9

Standardized Coefficients for a Measurement Model Consisting of Six Correlated Factors for the Entire Sample, and for Participants Between 18 and 49 Years of Age and Between 50 and 89 Years of Age

\begin{tabular}{|c|c|c|c|c|}
\hline & $\begin{array}{c}\text { All } \\
(N=330)\end{array}$ & $\begin{array}{c}18-49 \\
(n=181)\end{array}$ & & $\begin{array}{c}50-89 \\
(n=149)\end{array}$ \\
\hline \multicolumn{5}{|l|}{ Loadings of variables on constructs } \\
\hline $\mathrm{gF} \rightarrow$ Ravens & .86 & .81 & & .83 \\
\hline $\mathrm{gF} \rightarrow$ Letter sets & .71 & .67 & & .68 \\
\hline $\mathrm{gF} \rightarrow$ Analytical reasoning & 67 & .60 & & .63 \\
\hline $\mathrm{gF} \rightarrow$ Spatial relations & .82 & .84 & & .82 \\
\hline $\mathrm{gF} \rightarrow$ Paper folding & .82 & .83 & & .80 \\
\hline $\mathrm{gF} \rightarrow$ Form boards & .72 & .70 & & .66 \\
\hline Memory $\rightarrow$ Logical memory & .72 & .68 & & .77 \\
\hline Memory $\rightarrow$ Free recall & .81 & .76 & $<$ & .80 \\
\hline Memory $\rightarrow$ Paired associates & .69 & .74 & & .62 \\
\hline Speed $\rightarrow$ Letter comparison & .71 & .66 & & .76 \\
\hline Speed $\rightarrow$ Pattern comparison & .73 & .70 & & .64 \\
\hline Speed $\rightarrow$ Digit symbol & .85 & .74 & & .85 \\
\hline Vocab $\rightarrow$ WAIS III vocabulary & .86 & .86 & & .90 \\
\hline Vocab $\rightarrow$ WJ Picture vocabulary & .80 & .81 & & .77 \\
\hline Vocab $\rightarrow$ Antonym vocabulary & .89 & .88 & & .87 \\
\hline Vocab $\rightarrow$ Synonym vocabulary & .91 & .89 & & .89 \\
\hline Exec $\rightarrow$ Sort recognition & .68 & .57 & & .71 \\
\hline Exec $\rightarrow$ Letter fluency & .57 & .64 & & .60 \\
\hline Exec $\rightarrow$ Category fluency & .68 & .68 & & .72 \\
\hline Exec $\rightarrow$ Alternating fluency & .49 & .45 & & .51 \\
\hline Exec $\rightarrow$ Connections difference & .48 & .39 & & .50 \\
\hline Exec $\rightarrow$ Proverb interpretation & .43 & .39 & & .41 \\
\hline ProsMem $\rightarrow$ RedPen & .55 & .23 & $<$ & .55 \\
\hline ProsMem $\rightarrow$ RunMem & .59 & .42 & $<$ & .68 \\
\hline ProsMem $\rightarrow$ DrawClass & .52 & .37 & $<$ & .53 \\
\hline ProsMem $\rightarrow$ ConcptID & .44 & .37 & & .51 \\
\hline \multicolumn{5}{|l|}{ Correlations among constructs } \\
\hline $\mathrm{gF} \leftrightarrow$ Memory & .64 & .59 & & .59 \\
\hline $\mathrm{gF} \leftrightarrow$ Speed & .72 & .55 & & .71 \\
\hline $\mathrm{gF} \leftrightarrow$ Vocab & .33 & .56 & & .55 \\
\hline Memory $\leftrightarrow$ Speed & .62 & .43 & & .66 \\
\hline Memory $\leftrightarrow$ Vocab & .43 & .63 & & .57 \\
\hline Speed $\leftrightarrow$ Vocab & .09 & .17 & $<$ & .48 \\
\hline Exec $\leftrightarrow \mathrm{gF}$ & .76 & .65 & & .79 \\
\hline Exec $\leftrightarrow$ Memory & .79 & .74 & & .76 \\
\hline Exec $\leftrightarrow$ Speed & .78 & .61 & $<$ & .88 \\
\hline Exec $\leftrightarrow$ Vocab & .47 & .63 & & .62 \\
\hline ProsMem $\leftrightarrow$ Exec & .74 & .51 & & .80 \\
\hline ProsMem $\leftrightarrow \mathrm{gF}$ & .69 & .49 & & .73 \\
\hline ProsMem $\leftrightarrow$ Memory & .74 & .41 & & .84 \\
\hline ProsMem $\leftrightarrow$ Speed & .63 & .14 & $<$ & .73 \\
\hline ProsMem $\leftrightarrow$ Voc & .22 & .39 & & .47 \\
\hline
\end{tabular}

Note-All values are significantly $(p<.01)$ different from 0 except for those in bold. The " $<$ " sign indicates that the unstandardized coefficient for the 50- to 89-year-old group was significantly $(p<.01)$ larger than that for the 18- to 49-year-old group.

age to the prospective memory construct, corresponding to the dotted line in Figure 1, was - .33. However, because the cognitive abilities were all moderately correlated with one another (cf. Table 9), the estimates of the influence of each ability were distorted by multicollinearity. Separate analyses were therefore conducted in which each ability served as the only mediator of the age-related effects on the prospective memory construct.

Results of these analyses are presented in Table 10. The top row in the table contains the simple correlation between age and prospective memory. The remaining entries are organized into groups according to cognitive abilities, with the first row in each group containing the standardized coefficient for the relation of age to the ability, the second row containing the standardized coefficient for the relation of the ability to the prospective memory construct, and the third row containing the standardized coefficient for the direct relation of age to the prospective memory construct after taking into consideration the indirect effect through the ability (i.e., the semipartial correlation). Although the earlier analyses revealed that the variables used to assess executive functioning in this study had only weak construct validity, this construct was still included in the mediational analyses because it has been hypothesized to be closely linked to prospective memory.

Inspection of the table indicates that with the exception of when vocabulary ability served as the mediator, the direct or unique age-related effects on prospective memory were all much smaller than the total age-related effects. It can therefore be concluded that there was substantial statistical mediation of the age-related effects on the prospective memory construct through the age-related effects on other cognitive variables. However, with each ability there were still significant unique negative agerelated effects on the prospective memory construct in the data from the complete sample, and in the data for adults between 50 and 89 years of age.

The analyses just described were repeated using data only from participants with correct recall of the task instructions in all four prospective memory tasks $(N=$ 180). The results of these analyses are summarized in the bottom of Table 10, where it can be seen that, at least for adults over the age of 50, they are generally similar to those from the analyses based on all of the participants. The findings summarized in Table 10 therefore lead to the conclusion that although there is considerable mediation of the age-related effects through effects on other cognitive abilities, there also appear to be some unique, in the sense of statistically independent, age-related effects on the prospective memory construct, especially for adults over the age of 50 .

\section{Correlations Between Prospective Memory and Noncognitive Factors}

The final set of analyses consisted of using a structural equation model with the five cognitive ability constructs and the prospective memory construct all related to one another, and to a target variable representing either a personality trait or a self-rated memory factor. The latter variables were summed composite scores based on the factor analyses conducted by the original researchers (i.e., Costa \& McCrae, 2003; Zelinski et al., 1990) and based on groupings of items that were added to the MFQ. The relevant correlations are summarized in Table 11.

There are three interesting patterns in Table 11. One is that the personality trait of openness was positively correlated with all of the cognitive constructs except prospec- 
Table 10

Standardized Coefficients for Selected Paths in the Structural Model Portrayed in Figure 1 for the Entire Sample and for Participants Between 18 and 49 Years of Age and Between 50 and 89 Years of Age

\begin{tabular}{lcrrr}
\hline & All & $18-49$ & & $50-89$ \\
& $(N=330)$ & $(n=181)$ & & $(n=149$ \\
\hline Age $\rightarrow$ ProsMem & -.59 &. $\mathbf{0 9}$ & $>$ & -.73 \\
Age $\rightarrow$ Exec & -.43 &.$- \mathbf{1 1}$ & $>$ & -.54 \\
Exec $\rightarrow$ ProsMem & .62 & .49 & & .63 \\
Age $\rightarrow$ ProsMem [Exec] & -.33 &. $\mathbf{0 8}$ & $>$ & -.39 \\
Age $\rightarrow$ gF & -.61 & -.35 & & -.56 \\
gF $\rightarrow$ ProsMem & .58 & .52 & & .52 \\
Age $\rightarrow$ ProsMem [gF] & -.25 &. $\mathbf{2 3}$ & & -.45 \\
Age $\rightarrow$ Memory & -.47 & -.21 & $>$ & -.57 \\
Memory $\rightarrow$ ProsMem & .64 & .45 & $<$ & .69 \\
Age $\rightarrow$ ProsMem [Memory] & -.28 &. $\mathbf{1 3}$ & $>$ & -.33 \\
Age $\rightarrow$ Speed & -.70 & -.44 & & -.65 \\
Speed $\rightarrow$ ProsMem & .61 &. $\mathbf{0 7}$ & $<$ & .62 \\
Age $\rightarrow$ ProsMem [Speed] & -.17 &. $\mathbf{1 5}$ & $>$ & -.34 \\
Age $\rightarrow$ Vocabulary & .31 & .21 & &.$- \mathbf{0 4}$ \\
Vocabulary $\rightarrow$ ProsMem & .48 & .38 & & .47 \\
Age $\rightarrow$ ProsMem [Vocabulary] & -.74 &.$- \mathbf{0 3}$ & $>$ & -.71
\end{tabular}

Only Participants with Correct Recall of Prospective Memory Instructions in All Four Tasks

\begin{tabular}{|c|c|c|c|c|}
\hline \multicolumn{4}{|c|}{$(N=180)(n=109)$} & $(n=71)$ \\
\hline Age $\rightarrow$ ProsMem & -.25 & .11 & $>$ & -.60 \\
\hline Age $\rightarrow$ Exec & -.28 & -.18 & & -.13 \\
\hline Exec $\rightarrow$ ProsMem & .67 & .50 & & .49 \\
\hline Age $\rightarrow$ ProsMem $[$ Exec $]$ & -.07 & .09 & & -.55 \\
\hline Age $\rightarrow \mathrm{gF}$ & -.47 & -.35 & & -.39 \\
\hline $\mathrm{gF} \rightarrow$ ProsMem & .65 & .50 & & .63 \\
\hline Age $\rightarrow$ ProsMem $[\mathrm{gF}]$ & .04 & .18 & & -.32 \\
\hline Age $\rightarrow$ Memory & -.34 & -.24 & & -.48 \\
\hline Memory $\rightarrow$ ProsMem & .61 & .50 & & .73 \\
\hline Age $\rightarrow$ ProsMem [Memory] & -.05 & .10 & & -.27 \\
\hline Age $\rightarrow$ Speed & -.63 & -.45 & & -.55 \\
\hline Speed $\rightarrow$ ProsMem & .50 & -.02 & $<$ & .63 \\
\hline Age $\rightarrow$ ProsMem [Speed] & .06 & .01 & & -.25 \\
\hline Age $\rightarrow$ Vocabulary & .35 & .21 & & -.01 \\
\hline Vocabulary $\rightarrow$ ProsMem & .56 & .40 & & .66 \\
\hline Age $\rightarrow$ ProsMem [Vocabulary] & -.45 & -.11 & & -.63 \\
\hline
\end{tabular}

Note-All values are significantly $(p<.01)$ different from 0 except for those in bold. The " $>$ " or " $<$ " sign indicates that the unstandardized coefficient for the 50- to 89-year-old group differed significantly ( $p<$ .01 ) from that for the 18- to 49-year-old group.

tive memory. The second interesting pattern is that more favorable self-ratings of memory were associated with higher levels of most cognitive constructs. The fact that the correlations were similar in magnitude for all constructs, and were not higher for the episodic memory construct is somewhat surprising and raises the possibility that self-reports of memory may reflect aspects related to general self-efficacy, rather than something specific to memory. Finally, the third and most important point to note from Table 11 is that the pattern of correlations involving the prospective memory construct is generally similar to that with other constructs, which suggests that prospective memory does not have any stronger relations to these noncognitive factors than do other cognitive constructs.

\section{DISCUSSION}

Four questions motivated the present research. The first question was whether prospective memory could be considered a distinct construct, as reflected by evidence of both convergent and discriminant validity. The results of this study indicate that prospective memory can be assessed reliably in four different tasks that can be hypothesized to assess memory to perform actions in the future, and that the variables tend to represent a common dimension of individual differences. The relations among the variables were not very strong, particularly among adults under the age of 50, but they were significantly greater than zero in all but one case, and thus there is evidence of convergent validity. Furthermore, the prospective memory construct is distinct from constructs representing other cognitive abilities because the common variance shared by the prospective memory variables was only moderately related to other cognitive ability constructs, which suggests that the construct possesses discriminant validity. However, because the variables used to assess episodic memory were all based on tasks involving words or stories, it is possible that relations between prospective memory and episodic memory would have been stronger, and the evidence for discriminant validity weaker, if a broader variety of variables had been used to assess episodic memory.

Although the present results indicate that it is meaningful to refer to prospective memory as something different from episodic memory and other cognitive abilities, one must recognize that the standardized regression coefficients for the prospective memory variables in Table 9 were relatively small, with an overall median of .51. This indicates that an average of only about $25 \%$ of the variance in each variable was associated with what the four variables had in common. These values are weaker than those with variables used to represent other cognitive abilities and suggest that unlike the case with these other constructs, any single variable may assess only a limited aspect of the prospective memory construct. Researchers should therefore be cautious in drawing inferences about the construct of prospective memory on the basis of results from single variables.

Because the participants in this study performed several tests hypothesized to assess executive functioning, the construct validity of an executive functioning construct was also evaluated. Despite a different combination of variables, the conclusion from the four-model analysis of executive functioning in this study is similar to that reached by Salthouse et al. (2003) — namely, that variables used to assess executive functioning have weak to nonexistent construct validity. In the present study, only the three variables derived from fluency tests were significantly related to one another when the variables were allowed to be related to other cognitive abilities. Furthermore, when the variance each variable shared with four other cognitive abilities was controlled, none of the hypothesized executive functioning variables had 
Table 11

Correlations of Self-Rated Memory and Personality Dimensions with Cognitive Ability Constructs

\begin{tabular}{lccccccc}
\hline & Exec & $\mathrm{gF}$ & Mem & Speed & Voc & ProsMem \\
\hline Memory Self-Ratings & & & & & & \\
Global rating & $.22^{*}$ & $.21^{*}$ & $.20^{*}$ & $.25^{*}$ & .03 & $.17^{*}$ \\
Specific problems & $.19^{*}$ & $.20^{*}$ & .12 & $.20^{*}$ & .10 & .07 \\
General forgetting & $.26^{*}$ & $.20^{*}$ & $.19^{*}$ & $.24^{*}$ & $.19^{*}$ & $.15^{*}$ \\
Book forgetting & $.31^{*}$ & $.25^{*}$ & $.30^{*}$ & $.26^{*}$ & $.28^{*}$ & $.27^{*}$ \\
News forgetting & $.30^{*}$ & $.28^{*}$ & $.27^{*}$ & $.26^{*}$ & $.27^{*}$ & $.35^{*}$ \\
Memory over time & $.16^{*}$ & .02 & .11 & .08 & .09 & .09 \\
Seriousness & .06 & -.06 & -.01 & .04 & .09 & .05 \\
Retrospective rating & .09 & $.15^{*}$ & .09 & $.17^{*}$ & -.05 & -.01 \\
Use of mnemonics & .02 & .07 & -.11 & .06 & $-.13^{*}$ & -.09 \\
Personality Traits & & & & & & \\
Neuroticism & -.12 & $-.13^{*}$ & .02 & -.03 & $-.14^{*}$ & -.08 \\
Extraversion & .08 & .09 & -.02 & $.16^{*}$ & $-.18^{*}$ & .13 \\
Openness & $.30^{*}$ & $.20^{*}$ & $.29 *$ & $.19^{*}$ & $.31^{*}$ & .08 \\
Agreeableness & $.16^{*}$ & .01 & $.16^{*}$ & .06 & $.15^{*}$ & $.16^{*}$ \\
Conscientiousness & .06 & .05 & .05 & .10 & .04 & .13 \\
\hline
\end{tabular}

Note-Exec, executive functioning construct; $\mathrm{gF}$, fluid intelligence construct; Mem, episodic memory construct; speed, perceptual speed construct; Voc, vocabulary construct; ProsMem, prospective memory construct. For the memory self-ratings, higher scores indicate better self-ratings, less frequent problems, lower levels of perceived seriousness, improved memory over time, and less frequent reliance on memory aids. For personality traits, higher scores indicate higher values of the trait. $\quad * p<.01$.

significant residual variance in common. To the extent that a consistent pattern of individual differences is considered evidence of construct validity, these results suggest that variables based on various fluency tests, a proverb interpretation test, a sort recognition test, and a modified version of the trail making test lack validity in the sense that they do not appear to measure the same construct.

The second major question addressed in this study concerned the relation between a prospective memory construct and constructs representing episodic memory and executive functioning. As indicated in Tables 8 and 9 , both of these correlations were .74 . The correlation with the episodic memory construct is not surprising because both prospective memory and retrospective memory involve some of the same requirements to preserve information over time. The relation with the executive functioning construct is consistent with several earlier speculations (e.g., Mäntylä, 2003; McDaniel et al., 1999; Vogels et al., 2002), but this relation should nevertheless be interpreted cautiously because as just mentioned, the executive functioning construct did not have much construct validity. Furthermore, the correlations were nearly as large between the prospective memory construct and the $\mathrm{gF}$ and speed constructs, and thus the linkage between prospective memory and executive functioning may not be unique.

The third question motivating this research was the relation of the prospective memory construct to noncognitive factors such as self-perceptions of memory and dimensions of personality. The correlations in Table 11 indicate that prospective memory was significantly related to a number of factors derived from the self-ratings of memory, and with a personality dimension. However, it is important to note that other cognitive constructs also had similar relations to the memory ratings and to personality dimensions, and thus prospective memory does not appear to be unique with respect to its relations with noncognitive factors. The lack of stronger relations between the prospective memory construct and self-ratings of memory could be attributable to most of the questions in the memory questionnaire referring to retrospective, rather than prospective, memory. ${ }^{4}$

An interesting finding with respect to the memory ratings was that their relations were nearly as strong to other cognitive abilities as they were to episodic memory ability. These results lead to questions about the degree to which the ratings reflect specific experience with memory as opposed to subjective impressions about cognitive functioning more generally.

There was a consistent pattern for people higher on the personality trait of openness to perform at higher levels on each cognitive ability, with the notable exception of prospective memory. Relations between cognitive ability and the personality characteristic of openness have been reported before (e.g., Ackerman \& Heggestad, 1997; Ashton, Lee, \& Vernon, 2000) and appear to reflect a tendency for higher functioning individuals to be more intellectually curious and flexible.

The fourth question of interest in this study was the degree to which age-related effects on prospective memory were unique and statistically independent of the agerelated influences on other cognitive abilities. Because the latent constructs corresponding to $\mathrm{gF}$, episodic memory, perceptual speed, and executive functioning abilities all had moderately large negative correlations with age (cf. Table 10), the age-related effects on prospective memory might be of limited interest if they were simply a consequence of age-related effects on these other abilities.

Before considering the results relevant to the uniqueness of age-related influences on prospective memory, it is important to point out that age effects on the prospective memory construct were nonlinear and significantly different from zero only in adults over the age of 50. Unlike the abilities of gF, memory, and speed, in which significant negative age relations were apparent in adults between 18 and 49 years of age, the correlation between age and the prospective memory construct was not significantly different from zero for adults under the age of 50 , and in fact was slightly positive. As noted above, the lack of age sensitivity among the younger adults may be at least partially attributable to a measurement ceiling effect because the variances and reliabilities were substantially lower for adults under the age of 50 compared with adults between 50 and 89 years of age. Regardless of whether a measurement ceiling was responsible for all of the nonlinear effects, however, their existence in this study means that statements about age effects apply primarily to adults over the age of 50 .

The results in Table 10 indicate that although the relations of age to prospective memory are substantially re- 
duced after controlling for the variance in other cognitive abilities, in most cases the age-related variance was still significantly different from zero. Similar patterns were evident after controlling for each ability construct, including the episodic memory and executive functioning constructs. These results therefore suggest that some of the age-related effects on the prospective memory construct are unique, and specific to that construct, rather than being shared with age-related effects on other cognitive constructs.

A key question in light of the discovery of significant unique age-related influences on prospective memory is: What is the nature of those influences? Because processes of planning and scheduling were not included in the present executive functioning construct, it is possible that they may be involved in the statistically independent effects associated with age. Another possibility is that, as suggested by earlier theorists, good performance on prospective memory tasks may reflect aspects of personality or other noncognitive factors that were not assessed in this study.

In summary, the results of this study have demonstrated that prospective memory can be assessed reliably in several different tasks, and that as expected if the tasks involve a common dimension of individual differences, measures of performance on those tasks are moderately intercorrelated. The common variance among these prospective memory tasks was moderately correlated with constructs corresponding to other cognitive abilities, but not to such a degree that the constructs could be considered equivalent. In this respect, the variables used in this study to assess prospective memory exhibit construct validity. However, this was not the case for variables hypothesized to represent executive functioning because they were not significantly related to one another after controlling for the variance shared with other cognitive abilities. Finally, the prospective memory construct did not have any stronger relations to dimensions of personality or to self-ratings of memory than did other cognitive constructs, but it does appear to have some unique relations to age that are statistically independent of the age-related influences on other cognitive constructs.

\section{REFERENCES}

Ackerman, P. L., \& Heggestad, E. D. (1997). Intelligence, personality, and interests: Evidence for overlapping traits. Psychological Bulletin, 121, 219-245.

Ashton, M. C., Lee, K., \& Vernon, P. A. (2000). Fluid intelligence, crystallized intelligence, and the openness/intellect factor. Journal of Research in Personality, 34, 198-207.

Bennett, G. K., Seashore, H. G., \& Wesman, A. G. (1997). Differential Aptitude Test. San Antonio: Psychological Corporation.

Chasteen, A. L., PARK, D. C., \& Schwarz, N. (2001). Implementation intentions and facilitation of prospective memory. Psychological Science, 12, 457-461.

CHERRY, $\overline{\mathrm{K}}$. E., \& LeCompte, D. C. (1999). Age and individual differences influence prospective memory. Psychology \& Aging, 14, 6076.

Cherry, K. E., Martin, R. C., Simmons-D'Gerolamo, S. S., PinkStOn, J. B., Griffing, A., \& Gouvier, W. D. (2001). Prospective remembering in younger and older adults: Role of the prospective cue. Memory, 9, 177-193.
Cockburn, J., \& SMith, P. T. (1991). The relative influence of intelligence and age on everyday memory. Journal of Gerontology: Psychological Sciences, 46, P31-P36.

Costa, P. T., \& McCrae, R. R. (2003). NEO-FFI (NEO Five-Factor Inventory). Lutz, FL: Psychological Assessment Resources.

Craik, F. I. M., \& Kerr, S. A. (1996). Prospective memory, aging, and lapses of intention. In M. Brandimonte, G. O. Einstein, \& M. A. McDaniel (Eds.), Prospective memory: Theory and applications (pp. 227-237); Mahwah, NJ: Erlbaum.

Crowder, R. G. (1996). Commentary: The trouble with prospective memory. A provocation. In M. Brandimonte, G. O. Einstein, \& M. A. McDaniel (Eds.), Prospective memory: Theory and applications (pp. 143-147). Mahwah, NJ: Erlbaum.

Delis, D. C., Kaplan, E., \& Kramer, J. H. (2001). Delis-Kaplan Executive Function System. New York: Psychological Corporation.

DobBs, A. R., \& ReEves, M. B. (1996). Prospective memory: More than memory. In M. Brandimonte, G. O. Einstein, \& M. A. McDaniel (Eds.), Prospective memory: Theory and applications (pp. 199-225). Mahwah, NJ: Erlbaum.

DobBs, A. R., \& Rule, B. G. (1987). Prospective memory and selfreports of memory abilities in older adults. Canadian Journal of Psychology, 41, 209-222.

Einstein, G. O., \& MCDAniel, M. A. (1996). Retrieval processes in prospective memory: Theoretical approaches and some new empirical findings. In M. Brandimonte, G. O. Einstein, \& M. A. McDaniel (Eds.), Prospective memory: Theory and applications (pp. 115-141). Mahwah, NJ: Erlbaum.

Einstein, G. O., Smith, R. E., McDaniel, M. A., \& Shaw, P. (1997). Aging and prospective memory: The influence of increased task demands at encoding and retrieval. Psychology \& Aging, 12, 479-488.

Ekstrom, R. B., French, J. W., Harman, H. H., \& Dermen, D. (1976). Manual for kit of factor-referenced cognitive tests. Princeton, NJ: Educational Testing Service.

ELLIS, J. A. (1996). Prospective memory or the realization of delayed intentions: A conceptual framework for research. In M. Brandimonte, G. O. Einstein, \& M. A. McDaniel (Eds.), Prospective memory: Theory and applications (pp. 1-22). Mahwah, NJ: Erlbaum.

Goschke, T., \& Kuhl, J. (1996). Remembering what to do: Explicit and implicit memory for intentions. In M. Brandimonte, G. O. Einstein, \& M. A. McDaniel (Eds.), Prospective memory: Theory and applications (pp. 53-91). Mahwah, NJ: Erlbaum.

Graf, P., UtTl, B., \& Dixon, R. A. (2002). Prospective and retrospective memory in adulthood. In P. G. N. Ohta (Ed.), Lifespan development of human memory (pp. 257-282). Cambridge, MA: MIT Press.

GRE: Practicing to take the general test (1994). Princeton, NJ: Educational Testing Service.

Groot, Y. C. T., Wilson, B. A., Evans, J., \& Watson, P. (2002). Prospective memory functioning in people with and without brain injury. Journal of the International Neuropsychological Society, $\mathbf{8}, 645-$ 654.

Henry, J. D., Macleod, M. S., Phillips, L. H., \& Crawford, J. R. (2004). A meta-analytic review of prospective memory and aging. Psychology \& Aging, 19, 27-39.

HUPPERT, F. A., JOHNSON, T., \& NICKSON, J. (2000). High prevalence of prospective memory impairment in the elderly and in early-stage dementia: Findings from a population-based study. Applied Cognitive Psychology, 14, S63-S81.

Kidder, D. P., Park, D. C., Hertzog, C., \& Morrell, R. W. (1997). Prospective memory and aging: The effects of working memory and prospective memory task load. Aging, Neuropsychology, \& Cognition, 4, 93-112.

Kiñsella, G., Murtagh, D., Landry, A., Homfray, K., Hammond, M., O'Beirne, L., Dwyer, L., Lamont, M., \& Ponsford, J. (1996). Everyday memory following traumatic brain injury. Brain Injury, 10, 499-507.

MÄNTYL̈̈, T. (2003). Assessing absentmindedness: Prospective memory complaint and impairment in middle-aged adults. Memory \& Cognition, 31, 15-25.

MÄNTYLÄ, T., \& NILSSON, L.-G. (1997). Remembering to remember in adulthood: A population-based study on aging and prospective memory. Aging, Neuropsychology, \& Cognition, $\underline{\mathbf{4}}, \underline{81-92}$. 
Martin, M., \& Schumann-Hengsteler, R. (2001). How task demands influence time-based prospective memory performance in young and older adults. International Journal of Behavioral Development, 25, 386-391.

MAYLOR, E. A. (1993). Aging and forgetting in prospective and retrospective memory tasks. Psychology \& Aging, 8, 420-428.

MaYlor, E. A. (1996a). Age-related impairment in an event-based prospective memory task. Psychology \& Aging, 11, 74-78.

MaYlor, E. A. (1996b). Does prospective memory decline with age? In M. Brandimonte, G. O. Einstein, \& M. A. McDaniel (Eds.), Prospective memory: Theory and applications (pp. 173-197). Mahwah, NJ: Erlbaum.

Maylor, E. A., Darby, R. J., Logie, R. H., Della Sala, S., \& Sмith, G. (2002). Prospective memory across the lifespan. In P. G. N. Ohta (Ed.), lifespan development of human memory (pp. 235-256). Cambridge, MA: MIT Press.

Maylor, E. A., Smith, G., Della Sala, S., \& Logie, R. H. (2002). Prospective and retrospective memory in normal aging and dementia: An experimental study. Memory \& Cognition, 30, 871-884.

McDaniel, M. A., Glitsky, E. L., Rubin, S. R., GuYnN, M. J., \& Routhieaux, B. C. (1999). Prospective memory: A neuropsychological study. Neuropsychology, 13, 103-110.

Park, D. C., HerTZOG, C., KiDDER, D. P., Morrell, R. W., \& MAYHORN, C. B. (1997). Effect of age on event-based and time-based prospective memory. Psychology \& Aging, 12, 314-327.

RADLOFF, L. S. (1977). The CES-D scale: A self-report depression scale for research in the general population. Applied Psychological Measurement, 1, 385-401.

RAVEN, J. (1962). Advanced Progressive Matrices, Set II. London: Lewis.

ReEse, C. M., \& Cherry, K. E. (2002). The effects of age, ability, and memory monitoring on prospective memory task performance. Aging, Neuropsychology \& Cognition, 9, 98-113.

RoEDIGER, H. L., III (1996). Commentary: Prospective memory and episodic memory. In M. Brandimonte, G. O. Einstein, \& M. A. McDaniel (Eds.), Prospective memory: Theory and applications (pp. 149-155). Mahwah, NJ: Erlbaum.

Salthouse, T. A. (1993). Speed and knowledge as determinants of adult age differences in verbal tasks. Journal of Gerontology: Psychological Sciences, 48, P29-P36.

Salthouse, T. A., AtKinson, T. M., \& Berish, D. E. (2003). Executive functioning as a potential mediator of age-related cognitive decline in normal adults. Journal of Experimental Psychology: General, 132, 566-594.

SALTHOUSE, T. A., \& BABCOCK, R. L. (1991). Decomposing adult age differences in working memory. Developmental Psychology, 27, 763 776.

Salthouse, T. A., \& Ferrer-Caja, E. (2003). What needs to be explained to account for age-related effects on multiple cognitive variables? Psychology \& Aging, 18, 91-110.

Salthouse, T. A., Fristoe, N., \& Rhee, S. H. (1996). How localized are age-related effects on neuropsychological measures? Neuropsychology, 10, 272-285.

Salthouse, T. A., Toth, J., Daniels, K., Parks, C., Pak, R., Wol-
BRETTE, M., \& Hocking, K. (2000). Effects of aging on the efficiency of task switching in a variant of the Trail Making Test. Neuropsychology, 14, 102-111.

Schmidt, I. W., Berg, I. J., \& Deelman, B. G. (2001). Prospective memory training in older adults. Educational Gerontology, 27, 455478.

Uttl, B., Graf, P., Miller, J., \& Tuokko, H. (2001). Pro- and retrospective memory in late adulthood. Consciousness \& Cognition, $\mathbf{1 0}$, 451-472.

Vogels, W. W. A., Dekker, M. R., Brouwer, W. H., \& De Jong, R. (2002). Age-related changes in event-related prospective memory performance: A comparison of four prospective memory tasks. Brain \& Cognition, 49, 341-362.

WECHSLER, D. (1997a). Wechsler Adult Intelligence Scale-Third Edition. San Antonio: Psychological Corporation.

WeChSLER, D. (1997b). Wechsler Memory Scale-Third Edition. San Antonio: Psychological Corporation.

West, R., \& Craik, F. I. M. (2001). Influences on the efficiency of prospective memory in younger and older adults. Psychology \& Aging, 16, 682-696.

Woodcock, R. W. \& Johnson, M. B. (1990). Woodcock-Johnson PsychoEducational Battery - Revised. Allen, TX: DLM.

Zelinski, E. M., Gilewski, M. J., \& Anthony-Bergstone, C. R. (1990). Memory Functioning Questionnaire: Concurrent validity with memory performance and self-reported memory failures. Psychology \& Aging, 5, 388-399.

\section{NOTES}

1. Accuracy in complying with the prospective memory instruction was .71, .74, and .78 in Sessions 1, 2, and 3, respectively, indicating that, if anything, performance was higher with a longer delay since the instruction.

2. Because of the large number of statistical comparisons and moderately large sample sizes, a .01 value was used to evaluate statistical significance.

3. For all of the prospective memory variables, the median was larger than the mean, and thus the distributions were negatively skewed. However, by taking the $\log$ of 1 minus the value of the variable, the results of the analyses after transforming the variables were nearly identical to those with the untransformed variables, and thus only the results with the original variables are reported.

4. Some of the questions in the questionnaire were relevant to prospective memory (e.g., how often do you forget an event, such as attending a party or having a visitor; forget to bring up an important point you had intended to mention during a conversation; forget appointments; going to the store and forgetting what you wanted to buy), but a composite score based only on these items also had a very low correlation (i.e., $r=-.03$ ) with the prospective memory construct.

(Manuscript received October 10, 2003; revision accepted for publication February 13, 2004.) 